\title{
Integridad y multifuncionalidad: un modelo conceptual para hacer efectiva la restauración de los bosques nativos a gran escala.
}

\author{
Christian Little ${ }^{1,2^{*}}$, Bastienne Schlegel ${ }^{1}$, Gerardo Vergara ${ }^{1}$ \& Mauro E. González ${ }^{2,3}$ \\ ${ }^{1}$ Instituto Forestal, Sede Los Ríos. Valdivia, Chile. clittle@infor.cl \\ ${ }^{2}$ Center for climate and resilience Research (CR2). Santiago, Chile \\ ${ }^{3}$ Facultad de Ciencias Forestales y Recursos Naturales, Universidad Austral de Chile \\ *Autor de correspondencia \\ DOI: https://doi.org/10.52904/0718-4646.2021.545 \\ Recibido: 05.11.2021; Aceptado 10.12.2021
}

\begin{abstract}
RESUMEN
Se propone un modelo conceptual con un nuevo enfoque para las iniciativas que apoyen procesos de restauración de los bosques nativos. A partir del establecimiento de un ensayo de restauración a escala operativa, el modelo propone integrar variables de estado de los bosques con sus funciones ecológicas, esto con el objetivo de determinar niveles de integridad que apoyen la definición de los objetivos de la restauración y los indicadores de multifuncionalidad. Asociado al modelo, distinguimos una serie de barreras y oportunidades en los ámbitos ambientales, socio-económicos y de aprendizaje, presentando para cada uno de ellos un conjunto de recomendaciones que puedan aportar a la materialización de nuevas iniciativas de restauración de bosques nativos a gran escala en Chile. Concluimos sobre la importancia de estos nuevos enfoques y la necesidad de incluirlos como acompañamiento a la política pública y las decisiones del sector privado.
\end{abstract}

\section{SUMMARY}

A conceptual model directed towards new approaches that support native forest restoration processes is proposed. Based on the establishment of a restoration trial at an operational scale, the model proposes integrating variables of the state of forests with its ecological functions to determine levels of integrity that support the definition of restoration objectives and the indicators of forest multifunctionality. Associated with the model, we distinguish a series of barriers or opportunities in the environmental, socio-economic and learning fields, presenting for each of them a set of recommendations that can contribute to the achievement of new initiatives for the restoration of native forests on a large scale in Chile. We conclude on the importance of these new approaches and the need to include them as an accompaniment to public policy and private sector decisions.

\section{INTRODUCCIÓN}

El reconocimiento de una nueva época geológica, denominada Antropoceno (Lof et al., 2019), en la que el ser humano ha sido precursor de fenómenos globales, tales como el cambio climático, es también una época en la que se ha alterado gran parte de los ecosistemas en el mundo, incluyendo transformaciones en los bosques naturales (Malhi et al., 2014). Acciones antrópicas, tales como el cambio de uso de la tierra, la sobreexplotación de los bosques para la obtención de madera, así como formas menos visibles, como la ganadería, caza y la extracción de leña y carbón, han alentado a los países a suscribir numerosos acuerdos internacionales ${ }^{8}$ para frenar e incluso revertir los impactos de dichas acciones sobre los ecosistemas (Mekhlouf, 2021).

La reducción de beneficios para la sociedad, derivada de los impactos previamente señalados, ha sido el principal argumento para iniciar procesos de restauración (Mast et al., 1999; Fiedler, 2000; Arno y Fiedler, 2005) que recuperen la estructura y composición histórica, así como la función ecológica de los ecosistemas. En el caso de los bosques nativos en Chile, la pérdida y fragmentación de los mismos

${ }^{8}$ Convención Marco de las Naciones Unidas sobre el Cambio Climático (CMNUCC) para la Reducción de Emisiones por Deforestación y Degradación de los Bosques (REDD +), la Agenda 2030 de las Naciones Unidas para el Desarrollo Sostenible, así como metas en iniciativas de restauración global, incluido el Desafío de Bonn y la Declaración de Nueva York sobre los Bosques, que buscan restaurar 350 millones de hectáreas de tierras deforestadas y degradadas del mundo, así como reducir las emisiones de carbono para 2030. 
(Miranda et al., 2016; Vergara et al., 2019), junto con cambios en los regímenes de incendios (Sarricolea \& Úbeda, 2016; Gonzalez et al., 2018; González et al., 2020) o hidrológicos (Álvarez et al., 2020), se constituyen en importantes elementos que afectan la resiliencia de estos ecosistemas, y proyectan serias incertidumbres sobre los efectos en el largo plazo (Reyer et al., 2015).

La degradación de los bosques por presiones antrópicas y sus consecuentes efectos negativos en su funcionamiento y generación de servicios ecosistémicos (Vergara et al., 2019), hacen que preguntas tales como: ¿Cuál es el grado de daño o deterioro?, ¿a qué nivel el daño ha afectado y afectará los distintos componentes y procesos ecológicos?, ¿cómo será la estructura y composición en el mediano y largo plazo?, o ¿qué beneficios se deberán promover a largo plazo, considerando las cambiantes condiciones ambientales y sociales?, aún no tengan una respuesta clara para orientar las actividades de manejo y conservación, así como las acciones y políticas efectivas para la restauración de bosques a múltiples escalas.

\section{INTEGRIDAD Y MULTIFUNCIONALIDAD DE LOS BOSQUES NATIVOS}

De acuerdo a Parrish et al. (2003), la integridad es esencialmente el grado en que un sistema está libre de modificaciones significativas en cuanto a estructura, composición y funciones. Si bien este concepto ha recibido menos atención que otros directamente asociados a los beneficios para el ser humano que se derivan de los bosques (ej., servicios ecosistémicos (Bishop \& Landell-Mills, 2002; Brockerhoff et al., 2017), resulta ser un concepto clave para entender e identificar el nivel de daño o alteración del bosque, así como sus objetivos de multifuncionalidad, entendidos como la habilidad de los ecosistemas para proveer simultáneamente múltiples bienes y servicios (Manning et al., 2018).

En términos generales, un bosque con un bajo nivel de degradación (sin una alteración antrópica significativa), es decir, con una alta integridad, brindaría niveles más altos de beneficios, como el secuestro y almacenamiento de carbono, provisión de agua, uso forestal tradicional, contribución a los procesos climáticos locales y regionales y biodiversidad (Grantham et al., 2020). Sin embargo, para bosques dañados o alterados del mismo tipo, desconocer el grado de deterioro es una limitante, tanto para evaluar las propiedades físicas del ecosistema, como para lograr acuerdos respecto a qué se está perdiendo y qué es lo que se requiere recuperar a través de la restauración (Putz \& Redford, 2010). Es decir, qué se desea restaurar y para qué.

Tradicionalmente se considera la utilización de un ecosistema histórico para definir los objetivos y establecer una referencia, sin embargo, este enfoque está siendo reevaluado debido a las condiciones impuestas por el Antropoceno (Lof et al., 2019), siendo la multifuncionalidad el concepto que podría permitir orientar el manejo hacia la integración de las demandas sociales (Borras et al., 2016). De esta manera, considerando que la restauración se concibe como una actividad intencional que asiste o acelera la recuperación de un ecosistema respecto de su integridad y sostenibilidad (Gann et al., 2019), resulta entonces clave identificar cuáles son los objetivos que persigue la restauración, tanto para evaluar la respuesta del ecosistema, como para establecer los beneficios que de él se obtengan en el tiempo.

A partir de una experiencia práctica de restauración en bosques nativos adultos ${ }^{9}$, presentamos el siguiente modelo conceptual que integra las variables de estado que caracterizan la composición y estructura del bosque con las funciones ecológicas (Figura 1), a partir de las cuales es posible definir los objetivos que persigue la restauración y los indicadores de multifuncionalidad (Cuadro 1). Así, a las variables de estado que representan el espacio discreto entre todos los posibles estados y su trayectoria en el tiempo (Figura 1a), sería posible distinguir niveles de integridad a partir de la valoración actual y potencial de las funciones demandadas del bosque (Figura 1b), permitiendo a la vez compensaciones entre beneficios y funciones divergentes durante el proceso de restauración. Así mismo, tanto las variables de estado, como la funcionalidad ecológica pueden ser monitoreadas en el tiempo para el logro de acuerdos en cuanto a los beneficios que deben priorizarse.

\footnotetext{
${ }^{9}$ Ensayo de restauración a escala operativa bajo convenio INFOR-FORESTAL S.A. Ensayo desarrollado para bosques adultos del tipo forestal Coigüe-Raulí-Tepa ubicado en la comuna de Panguipulli. Se proyecta la restauración de 27 hectáreas.
} 


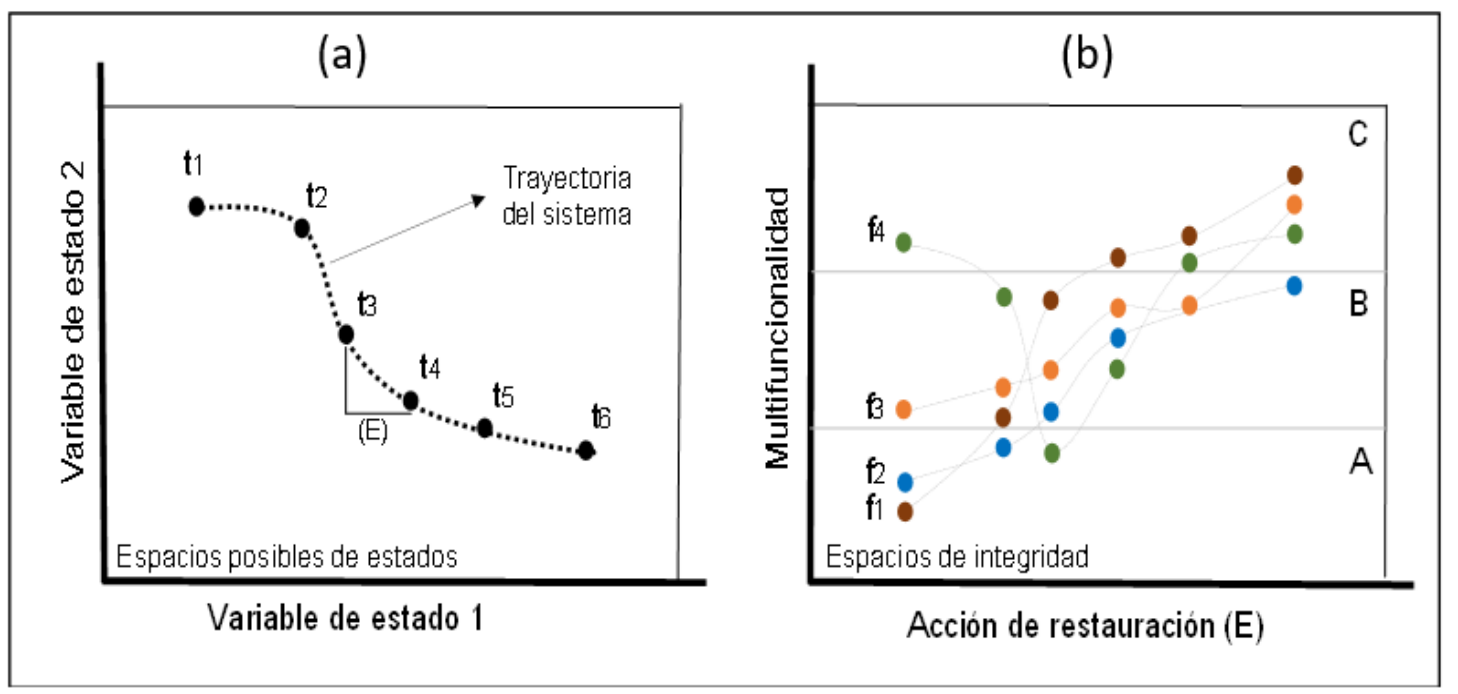

Figura 1. a) Espacio posible de estados respecto a variables de composición y estructura del bosque que cambian en el tiempo como consecuencia de acciones de restauración que implican incorporación de energía (E). Variables de estado, puede ser la relación de. número de árboles por hectárea y el área basal. b) Niveles de integridad (A, B y C) respecto a la identificación de las funciones (f) del ecosistema identificadas en el Cuadro 1.

Cuadro 1. Función ecológica, ejemplo de objetivos de restauración e indicadores de multifuncionalidad.

\begin{tabular}{llcl}
\hline \multicolumn{1}{c}{$\begin{array}{c}\text { Función } \\
\text { ecológica }\end{array}$} & $\begin{array}{c}\text { Objetivo de } \\
\text { restauración } \\
\text { (bienes y } \\
\text { servicios) }\end{array}$ & Indicador(es) de multifuncionalidad \\
\hline Provisión & (f1) & $\begin{array}{c}\text { Madera de } \\
\text { calidad }\end{array}$ & $\begin{array}{c}\text { Presencia y abundancia de especies de alto valor } \\
\text { de acuerdo al potencial de sitio. }\end{array}$ \\
\hline Información (f2) & $\begin{array}{c}\text { Investigación y } \\
\text { educación }\end{array}$ & Acciones de monitoreo y difusión de la ciencia. \\
\hline Soporte & (f3) & $\begin{array}{c}\text { Diversidad de } \\
\text { especies }\end{array}$ & Abundancia de especies vegetales y nichos. \\
\hline Regulación (f4) & $\begin{array}{c}\text { Secuestro de } \\
\text { carbono }\end{array}$ & Tasa de crecimiento del bosque. \\
\hline
\end{tabular}

Para poner en marcha este modelo propuesto, en el Cuadro 2 se identifican los principales desafíos/oportunidades asociadas a los objetivos de restauración, los cuales abarcan los componentes ambientales, socio-económicos y de aprendizaje, que permitan hacer efectiva la restauración (Lara et al., 2014).

Para cada desafío/oportunidad, presentamos un conjunto de recomendaciones orientado a la recuperación de la multifuncionalidad de los bosques, convirtiendo a la restauración en una oportunidad para incrementar la eficiencia de las acciones según decisiones guiadas por intereses y expectativas asociadas al suministro de bienes y servicios ecosistémicos. 
Cuadro 2. Principales promotores para iniciar procesos de restauración de bosques nativos sometidos a procesos de degradación y recomendaciones a ser implementadas. Para cada promotor se presenta los desafíos/amenazas (A) y oportunidades para la restauración (B)

\begin{tabular}{l} 
Promotores de Restauración \\
\hline \multicolumn{1}{c}{ Componentes amb } \\
\hline Degradación Forestal. \\
(A) Se estima que aproximadamente 450.000 hectáreas de \\
bosques nativos del centro sur de Chile podrían estar en una \\
condición de bosques degradados (Bahamondez \& \\
Thompson, 2016). Además, se desconoce la superficie de \\
bosques nativos en proceso de degradación forestal (Vergara \\
et al., 2019). \\
(B) Se considera a la restauración de bosque nativo como un \\
componente clave de la Política Forestal de Chile 2015-2035 \\
y el cumplimiento de los compromisos internacionales en \\
materia de conservación de la biodiversidad.
\end{tabular}

\section{Cambio Climático.}

(A) Las predicciones de cambio climático para en el centro-sur de Chile proyectan reducciones de precipitaciones y aumento de temperaturas (Boisier et al., 2016).

(B) Se considera a la restauración de bosque nativo como un componente clave de la Estrategia Nacional de Cambio Climático.

Recomendaciones

- Identificar métricas que permitan evaluar la integridad y multifuncionalidad de los bosques nativos acordes al potencial de sitio.

- Entender la relación de la integridad y multifuncionalidad con la provisión de bienes, servicios ecosistémicos y la biodiversidad.

- Identificar prioridades de restauración en base a la integridad y multifuncionalidad de los bosques nativos para la recuperación de beneficios ambientales y sociales.

\begin{tabular}{l}
\hline \multicolumn{1}{c}{ Componentes Socio } \\
\hline \multicolumn{1}{c}{ Promotores de Restauración } \\
\hline $\begin{array}{l}\text { Empleo para economías regionales y locales en sectores } \\
\text { rurales. }\end{array}$
\end{tabular}

(A) Necesidad de incrementar la efectividad en aplicación de los incentivos del Estado para el manejo y recuperación del bosque nativo (Schlegel et al., 2020).

(B) Creciente interés privado en recuperar y manejar bosques nativos en sitios de alta productividad. Interés público (Ministerio del Medio Ambiente y la Corporación Nacional Forestal) en la identificación, priorización y mapeo de áreas clave de restauración como una actividad que aporte a la economía regional y local (Altamirano et al., 2007). Interés público y privado para el establecimiento y promoción de programas de la producción de plantas de especies nativas y establecimiento de especies nativas con fines multipropósito (Bannister et al., 2018; Schlegel et al., 2020). Además, se busca establecer una política y planes para la restauración a gran escala (Bannister et al., 2018) para cumplir con el compromiso del país de recuperar 500.000 hectáreas de bosques propuestas por los objetivos de las Contribuciones Determinadas a Nivel Nacional (NDC).

\section{Mercado de Servicios Ecosistémicos.}

(A) Inexistencia de programas del Estado asociados a mecanismos de compensación ambiental relacionados a la oferta y demanda de servicios ecosistémicos de los bosques nativos (Little y Lara, 2014).

(B). Inicio de proyecto +Bosques (FAO-CONAF) enfocado al mercado del carbono y proyecto (MMA-GEF) enfocado a generar mecanismos de retribución económica por el servicio ecosistémico provisión de agua y conservación de la biodiversidad.

\begin{tabular}{ll}
\hline \multicolumn{1}{c}{ Componentes Aprendizaje } \\
\hline \multicolumn{1}{c}{ Promotores de Restauración } & \multicolumn{1}{c}{ Recomendaciones } \\
\hline Formación/Investigación. & - Incrementar el nivel de conocimiento e información \\
(A) carencia de personal capacitado en diferentes niveles de & $\begin{array}{l}\text { sobre la respuesta de los ecosistemas, costos y } \\
\text { beneficios asociados con las diferentes opciones de } \\
\text { formación (profesionales, practicantes, capataces, operarios) } \\
\text { restauración incluyendo sus diferentes componentes. }\end{array}$ \\
\hline
\end{tabular}

- Incorporar los avances conceptuales en las leyes y normas en materia de mitigación y adaptación al cambio climático.

- Incorporar los conceptos de degradación y restauración en los instrumentos de política forestal y ambiental.

- Proporcionar información a tomadores de decisión local y partes interesadas respecto a la integridad y multifuncionalidad de los bosques nativos.

\section{-Económicos}

\section{Recomendaciones}

- Adecuar la Ley 20.283 para incorporar subsidios específicos dirigidos a la restauración de bosques nativos con objetivos multifuncionales definidos.

Modificar planes de manejo acordes a la determinación de la integridad del bosques y objetivos multifuncionales definidos.

- Generar bases de datos y protocolos públicos y compartidos de desempeño inicial, crecimiento y productividad de los bosques.

- Identificar bosques remanentes locales para suministrar material genético para la propagación de plantas e incentivos asociados al uso de material genético local de especies nativas.

- Generar protocolos para la colecta de semilla y producción de plantas y disponer de información pública respecto a las labores silviculturales asociadas a desempeño de plántulas en la fase de establecimiento.

- Implementar programas de retribución ambiental en bosques con objetivos multifuncionales.

Determinar los costos e ingresos de faenas silvícolas a escala operativa asociadas a la restauración de bosques nativos con distinto grado de alteración y objetivos de multifuncionalidad. 


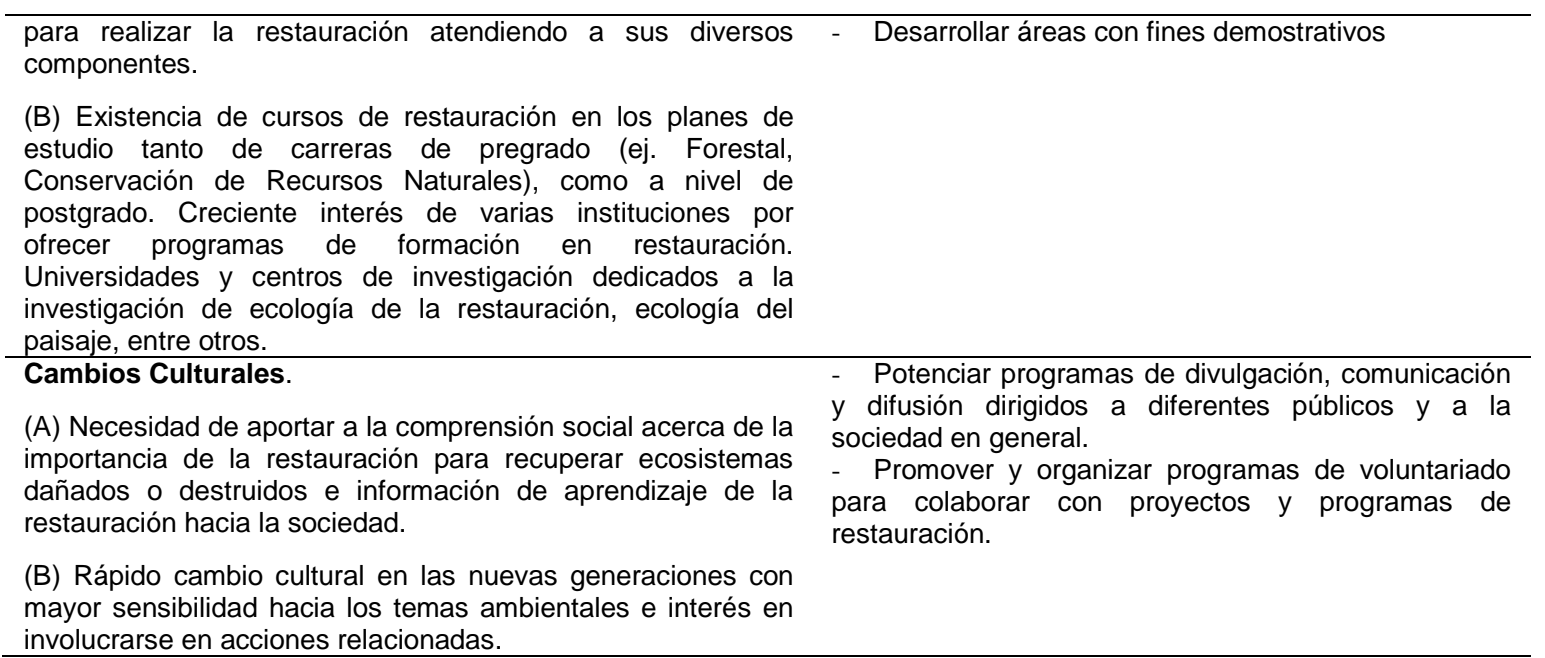

\section{CONCLUSIONES}

En los últimos 50 años ha habido notables avances respecto del conocimiento sobre ecología y dinámica de bosques nativos en Chile, así como los beneficios sociales que representan estos ecosistemas para el bienestar humano. Sin embargo, la huella asociada al uso histórico de los bosques, representa una barrera para su conservación y manejo en aquellas áreas donde los bosques han sobrepasado umbrales de cambio hacia condiciones menos favorables, y requieren de atención por parte del Estado debido a su importancia ecológica, productiva o social.

El enfoque de integridad y multifuncionalidad de los bosques nativos representaría una oportunidad para identificar los objetivos de manejo y las métricas para su monitoreo en el tiempo. Este enfoque permitiría incorporar valores intangibles del bosque, considerado un recurso renovable en cuanto la tasa de crecimiento y mantención de funciones ecológicas sean acordes a la escala temporal asociada a la demanda por beneficios (Schlegel, 2020).

El enfoque de la integridad y multifuncionalidad, basado en la teoría y experiencia práctica actual, podría ser incorporado en el desarrollo de políticas públicas, como por ejemplo la Ley de Bosque Nativo, o las decisiones del sector privado, en reconocimiento al valor ecológico y social de éstos, haciendo efectiva la restauración a gran escala.

\section{REFERENCIAS}

Altamirano, A., Echeverría, C. \& Lara, A. (2007). Efecto de la fragmentación forestal sobre la estructura vegetacional de las poblaciones amenazadas de Legrandia concinna (Myrtaceae) del centro-sur de Chile. Revista Chilena de Historia Natural, 80. Pp: 27-42. https://doi.org/10.4067/S0716-078X2007000100003

Alvarez-Garreton, C. Boisier, J.P., Garreaud, R., Seibert, J. \& Vis, M. (2020). Progressive water deficits during multiyear droughts in central-south Chile. Hydrol. Earth Syst. Sci. Discuss., Pp: 1-23. https://doi.org/10.5194/hess-2020-249

Arno, S.F. \& Fiedler, C.E. (2005). Mimicking Nature's fire: Restoring fire-prone forests in the West. Island Press, Washington, DC.

Bahamondez, C. \& Thompson, I.D. (2016). Determining forest degradation, ecosystem state and resilience using a standard stand stocking measurement diagram: theory into practice. Forestry, 89(3): $290-300$. https://doi.org/10.1093/forestry/cpv052

Bannister, J.R., Vargas-Gaete, R., Ovalle, J.F., Acevedo, M., Fuentes-Ramirez, A., Donoso, P.J., Promis, A. et al. (2018). Major bottlenecks for the restoration of natural forests in Chile. Restoration Ecology, 26. Pp: 10391104. https://doi.org/10.1111/rec.12880 
Bishop, J. \& Landell-Mills, N. (2002) Forest environmental services: an overview. In: Pagiola, S., Bishop, J. \& LandellMills, N. (Eds). Selling Forest Environmental Services: Market-Based Mechanisms for Conservation and Development. Earthscan Publications. London. Pp: 15-35.

Boisier, J. P., Rondanelli, R., Garreaud, R., \& Muñoz, F. (2016). Anthropogenic and natural contributions to the Southeast Pacific precipitation decline and recent mega-drought in central Chile. Geophys. Res. Lett., 43(1): 413-421. https://doi.org/10.1002/2015GL067265.

Borrass L., Kleinschmit D. \& Winkel G. (2016). The "German model" of integrative multifunctional forest management-Analysing the emergence and political evolution of a forest management concept. Forest Policy and Economics, 77. Pp: 16-23. https://doi.org/10.1016/j.forpol.2016.06.028

Brockerhoff, E.G., Barbaro, L., Castagneyrol, B., Forrester, D.I., Gardiner, B., González-Olabarria, J.R., Lyver, P.O.B. et al. (2017). Forest biodiversity, ecosystem functioning and the provision of ecosystem services. Biodivers. Conserv., 26. Pp: 3005-3035. https://doi.org/10.1007/s10531-017-1453-2

Fiedler, C.E. (2000). Restoration treatments promote growth and reduce mortality of old-growth ponderosa pine (Montana). Ecol. Restoration, 18. Pp: 117-119.

González, ME., Gómez-González, S., Lara, A., Garreaud, R. \& Díaz-Hormazábal, I. (2018). The 2010-2015 Megadrought and its influence on the fire regime in central and south-central Chile. Ecosphere, 9(89): e02300. https://doi.org/10.1002/ecs2.2300

González, M.E., Sapiains, R., Gómez-González, S., Garreaud, R., Miranda, A., Galleguillos, M., Jacques, M. et al. (2020). Incendios forestales en Chile: causas, impactos y resiliencia. Centro de Ciencia del Clima y la Resiliencia (CR)2, Universidad de Chile, Universidad de Concepción y Universidad Austral de Chile.

Grantham, H.S., Duncan, A. \& Watson, E.M. (2020). Anthropogenic modification of forests means only $40 \%$ of remaining forests have high ecosystem integrity. Nat. Commun., 11. Art. $\mathrm{N}^{\circ} 5978$. https://doi.org/10.1038/s41467-020-19493-3

Gann, G.D., McDonald, T., Walder, B., Aronson, J., Nelson, C.R., Jonson, J., Hallett, J.G. et al. (2019). International principles and standards for the practice of ecological restoration. 2nd edition. Restoration Ecology, 27. Pp: S1-S46. https://doi.org/10.1111/rec.13035

Lara, A., Little, C., Cortés, M., Cruz, E., González, M., Echeverría, C., Suarez, J. et al. (2014). Restauración de ecosistemas forestales. En: Donoso, C., González, M.E. \& Lara, A. (Eds). Ecología Forestal: Bases para el Manejo Sustentable y Conservación de los Bosques Nativos de Chile. Ediciones Universidad Austral de Chile. Valdivia. $720 \mathrm{p}$.

Little, C. \& Lara, A. (2014). Servicios Ecosistémicos de los Bosques Nativos del Centro Sur de Chile. En: Donoso, C., González, M.E. \& Lara, A. (Eds). Ecología Forestal: Bases para el Manejo Sustentable y Conservación de los Bosques Nativos de Chile. Ediciones Universidad Austral de Chile. Valdivia. 720 p.

Löf, M., Madsen, P., Metslaid, M., Witzell, J. \& Jacobs, D.F. (2019). Restoring forests: regeneration and ecosystem function for the future. New Forests, 50. Pp: 139-151. https://doi.org/10.1007/s11056-019-09713-0

Malhi, Y., Gardner, T.A., Goldsmith, G.R. Silman, M.R. \&, Zelazowski, P. (2014). Tropical Forests in the Anthropocene. Annu. Rev. Environ. Resour. 39. Pp: 125-159. https://doi.org/10.1146/annurev-environ030713-155141

Manning, P., van der Plas, F., Soliveres, S., Allan, E., Maestre, F. T., Mace, G., Whittingham, M. et al. (2018). Redefining ecosystem multifunctionality. Nature Ecology \& Evolution, 2. Pp: 427-436. https://doi.org/10.1038/s41559-017-0461-7

Mast, J.N., Fulé, P.Z., Moore, M.M., Covington, W.W. \& Waltz, A.E.M. (1999). Restoration of presettlement age structure of an Arizona ponderosa pine forest. Ecological Applications, 9. Pp: 228-239. https://doi.org/10.1890/1051-0761(1999)009[0228:ROPASO]2.0.CO;2

Mekhlouf, O. (2021). International Legal protection of forest in the context of sustainable development. IOP Conf. Ser.: Earth Environ. Sci., 779 012131. https://doi.org/10.1088/1755-1315/779/1/012131

Miranda, A. Altamirano, A., Cayuela, L., Lara, A. \& González, M.E. (2016). Native forest loss in the Chilean biodiversity hotspot: revealing the evidence. Regional Environmental Change, 17. Pp: 285-297. https://doi.org/10.1007/s10113-016-1010-7

Parrish, J.D., Braun, D.P. \& Unnasch, R.S. (2003). Are we conserving what we say we are? Measuring ecological integrity within protected areas. BioScience, 53. Pp: 851-860. https://doi.org/10.1641/00063568(2003)053[0851:AWCWWS]2.0.CO;2 
Putz, F.E. \& Redford, K.H. (2010). The Importance of Defining 'Forest': Tropical Forest Degradation, Deforestation, Long-Term Phase Shifts, and Further Transitions. Biotropica, 42. Pp: 10-20. https://doi.org/10.1111/j.17447429.2009.00567.x

Reyer, C.P., Rammig, A., Brouwers, N. \& Langerwisch, F. (2015). Forest resilience, tipping points and global change processes. J. Ecol., 103. Pp: 1-4. https://doi.org/10.1111/1365-2745.12342

Schlegel, B., Little, C., Urrutia, M., Hernández, G. \& Pasten, R. (2020). Incorporando la multifuncionalidad en la evaluación económica de proyectos de restauración de bosques nativos siempreverdes en el sur de Chile. Ciencia \& Investigación Forestal, 26(1): 23-40. https://doi.org/10.52904/0718-4646.2020.525

Sarricolea, P. Úbeda, X. (2016). Wildfires in Chile: A review. Global and Planetary Change, 146. Pp: $152-161$. https://doi.org/10.1016/j.gloplacha.2016.10.004

Vergara, G., Schlegel, B., Little, C., Mujica, R. \& Martin, M. (2019). ¿Degradación o Degradado? Necesidad de una Propuesta Conceptual para Recuperar la Funcionalidad y Capacidad Productiva de los Bosques. Ciencia \& Investigación Forestal, 25(1): 69-79. https://doi.org/10.52904/0718-4646.2019.511 